\title{
Pathogenicity of three entomopathogenic fungi against the Mediterranean fruit fly, Ceratitis capitata (Wiedemann) (Diptera: Tephritidae)
}

\author{
N. A. Soliman', Sherihan M. Al-amin ${ }^{*}$, Amira E. Mesbah', Ahmed M. A. Ibrahim² and Ali M.A. Mahmoud²
}

\begin{abstract}
The use of pesticides against the Mediterranean fruit fly, Ceratitis capitata (Wiedemann) (Diptera: Tephritidae) as a tool to control the pest has become an obstacle to the fresh agricultural products export to many countries that restrict pesticides residues. The effectiveness of three local strains of entomopathogenic fungi: Metarhizium anisopliae, Beauveria bassiana, and Paecilomyces lilacinus against the adult and immature stages of C. capitata was evaluated under laboratory conditions. Obtained results showed that M. anisopliae and B. bassiana were superior in its pathogenicity and potential to kill the pest than P. lilacinus. These results may be important to be used for the control of the pest in IPM program.
\end{abstract}

Keywords: Ceratitis capitata, Entomopathogenic fungi, Pathogenicity, Fruit fly

\section{Background}

The Mediterranean fruit fly, Ceratitis capitata (Wiedemann) (Diptera: Tephritidae), is one of the economic horticultural pests in Egypt and Mediterranean basin due to its ability to infest wide range of fruits. Ceratitis capitata attacks more than 300 different hosts and leave negatively economic impact (Papadopoulos et al. 2001). Flies control depends mainly on chemical sprays (organophosphates, especially malathion, pyrethroids, and spinosad) mixed with protein baits (Martinez-Ferrer et al. 2012) in the orchards as well as larvae and pupae found in soil (Stark and Vargas 2009). However, synthetic pesticides for crop protection in aggravating use cause a number of undesirable effects on human health and the environment (Perry et al. 1998).

Entomopathogenic fungi (EPF) are soil-borne microorganisms, where optimal temperature in agricultural soil for their growth, sporulation, and infection often ranges

\footnotetext{
* Correspondence: tamersherihan@gmail.com

${ }^{1}$ Plant protection research institute, ARC, Dokki, Giza, Egypt

Full list of author information is available at the end of the article
}

from 20 to $30{ }^{\circ} \mathrm{C}$, but variation in temperature tolerance within a strain can be significant. There are several factors negatively affecting on EPF presence in the soil such as copper content and fungicides (Uzman et al. 2019), nevertheless, Metarhizium spp. can be the dominant EPF genus in agricultural soil (Thaczuk et al. 2014) than Beauveria bassiana (Balsamo) Vuillemin. Also, organic matter (for M. anisopliae (Metschnikoff) Sorokin) and $\mathrm{pH}$ clay content (for B. bassiana) did not affect the occurrence of EPF (Quesada-Moraga et al. 2007). Consequently, EPF form an important component of an integrated pest management strategy for fruit flies via soil treatment. Also, EPF had effective role in fly regulation in the field (Sookar 2014). Entomopathogenic fungi, compared to other soil micro-organisms, have the advantage that they can infect their hosts via contact, invade via the epicuticle of integument, and do not need to be ingested by the insect to cause infection (Lacey and Shapiro-Ilan 2008).

Many investigations revealed that EPF have a significant role as biological control agents against different 
tephritids (Soliman et al. 2014). Beauveria bassiana and M. anisopliae are highly virulent against the Mediterranean fruit fly and can infect adults, larvae, or pupae via different routes of exposure (Castillo and Moya 2000; Ekesi et al. 2002, 2003; Dimbi et al. 2003; Konstantopoulou and Mazomenos 2005; Quesada-Moraga et al. 2006).

The objective of this investigation was to evaluate the virulence of locally isolated strains of the EPF; $M$. anisopliae, B. bassiana, and Paecilomyces lilacinus against $C$. capitata stages via different concentrations under laboratory conditions.

\section{Material and methods}

\section{Elaboration of rearing}

The Mediterranean fruit fly C. capitata was obtained from the rearing laboratory of Horticultural Pest Department, Plant Protection Research Institute (PPRI), Agricultural Research Center (ARC), Giza, Egypt. Flies fed on sugar and protein hydrolysate in a ratio 3:1, respectively (ElSayed 1979), in addition to a source of water. Produced eggs were collected daily and reared on the artificial rearing medium described by Tanaka et al. (1969). After larvae complete the third larval instar, larvae were received in water then collected in fine sand obtained from Al-Arish City (Saini peninsula) for pupation. A day before the emergence of flies, pupae were sieved and located in screen cage $(30 \mathrm{~cm} \times 30 \mathrm{~cm} \times 30 \mathrm{~cm})$ allowing the emerged flies to feed, mate, and produce eggs.

\section{Fungal strains and conidiospores production}

Local strains of B. bassiana 5133, M. anisopliae 5130, and $P$. lilacinus 1062 were used in the current study. These isolates were obtained from the Center for Fungi, Faculty of Science, Assiut University, Assiut, Egypt. Conidial viability test was achieved on these isolates. Conidia obtained from the 3 fungal cultures were grown on potato dextrose agar (PDA), consisting of $10 \mathrm{~g} / \mathrm{l}$ potato, $20 \mathrm{~g} / \mathrm{l}$ glucose, and $20 \mathrm{~g} / \mathrm{l}$ agar-agar in Petri dishes $(9 \mathrm{~cm}$ diameter and $1.5 \mathrm{~cm}$ in height). After 15 days of incubation at $25{ }^{\circ} \mathrm{C}$ and $60 \% \mathrm{RH}$, the conidia were harvested from the plates by grazing with sterile spatula into $1 \%$ Tween-80. The suspension was vortexed for $30 \mathrm{~min}$ using agitator at room temperature. The conidial concentration of the resulting stock suspension was estimated using hemocytometer under light microscope for each fungus. A series of dilutions were made to give concentrations range of $10^{6}, 10^{7}, 10^{8}, 10^{9}$, and $10^{10}$ conidia/ml suspensions were held on ice at $4{ }^{\circ} \mathrm{C}$ to prevent conidial germination until using in bioassay as described by Gabarty et al. (2014).

\section{Bioassay tests}

Adults, third larval instar, and pupae were used in this bioassay. Ten third instar larvae were dipped in $2 \mathrm{ml}$ of the above-mentioned fungal concentrations (conidia/ml) for $30 \mathrm{~s}$, then transferred to a Petri dish containing 10 mg standard steam-autoclaved-sterilized fine sand, covered by the lid, sealed with plastic parafilm to avoid external contamination, and kept at $25{ }^{\circ} \mathrm{C}$ in a complete darkness. A plastic cage, $0.5 \mathrm{~L}$ capacity, was sprayed by 2 $\mathrm{ml}$ of conidial suspension and kept to dry, and then 10 newly emerged flies (unequal in sex) were entered. Control cages were treated by $2 \mathrm{ml}$ of $1 \%$ tween 80 . Artificial diet was supplied. Flies mortality were recorded 6 days after treatment. Dead flies were incubated in $25^{\circ} \mathrm{C}$ and $65 \% \mathrm{RH}$ to observe mycosis. All bioassays were recorded and analyzed based on four independent replicates.

\section{Statistical analysis}

Mortalities were corrected using Abbott formula (Abbott 1925). Probit analysis was used to determine lethal and sublethal levels (Finney 1971). The difference among treatments in rate of mortality was determined by analysis of variance (ANOVA) and Tukey's method was used to compare mean significant differences among treatments $(p<0.05)$ using SPSS 26 statistical software.

\section{Results and discussion}

This study addressed the pathogenicity of three EPF: $M$. anisopliae, B. bassiana, and P. lilacinus, against different stages of $C$. capitata, with different levels (Tables 1, 2, 3, and 4). To address the pathogenicity, it was needed to analyze the effect of different fungal concentrations on different immature stages of $C$. capitata as well as adult stage.

\section{Susceptibility of $C$. capitata larvae}

Mean percentage of larval mortality of $C$. capitata treated with different concentrations of fungi showed significant increase in mortality $(p$ value $<0.05)$ in a concentration-dependent manner (Table 1). The fungus $M$. anisopliae caused the highest percentages of larval mortality in late third larval instar that ranged $15-60 \%$ at the $1 \times 10^{6}$ and $1 \times 10^{10}$ conidia/ml, respectively. The mortality rate appeared to increase by increasing concentration. Similarly, B. bassiana ranked second recording (15-57.50\%) at the same concentrations like $M$. anisopliae. On the contrary, P. lilacinus caused the lowest percentage of larval mortality ranging $10-17.5 \%$. Interestingly, concentration increase in $P$. lilacinus treated larvae did not affect the mortality rate (Table 1).

To further select which of the tested fungi is high in pathogenicity, the differences in mortality among EPF at the same concentration were statistically analyzed (Fig. 1). The obtained results revealed no differences in larval sensitivity among the three EPF, at the lowest concentration, $1 \times 10^{6}$ (Fig. 1a). However, at high concentrations, 
Table 1 Susceptibility of Ceratitis capitata immature stages to Metarhizium anisopliae, Beauveria bassiana, and Paecilomyces lilacinus at different concentrations

\begin{tabular}{|c|c|c|c|c|c|}
\hline \multicolumn{6}{|c|}{ Mean $\%$ mortality of larvae } \\
\hline \multicolumn{6}{|c|}{ Metarhizium anisopliae } \\
\hline $\begin{array}{l}1 \times 10^{6} \\
\text { Conidia/ml }\end{array}$ & $\begin{array}{l}1 \times 10^{7} \\
\text { Conidia/ml }\end{array}$ & $\begin{array}{l}1 \times 10^{8} \\
\text { Conidia/ml }\end{array}$ & $\begin{array}{l}1 \times 10^{9} \\
\text { Conidia/ml }\end{array}$ & $\begin{array}{l}1 \times 10^{10} \\
\text { Conidia/ml }\end{array}$ & Control \\
\hline $15.00^{b} \pm 5.77$ & $27.50^{c} \pm 5.00$ & $37.50^{d} \pm 5.00$ & $50.00^{e} \pm 8.16$ & $60.00^{f} \pm 8.16$ & $5.00^{a} \pm 5.77$ \\
\hline$F=41.760$ & & $p$ value $=.000$ & & $\mathrm{df}=5,18$ & \\
\hline \multicolumn{6}{|c|}{ Beauveria bassiana } \\
\hline $15.00^{b} \pm 5.77$ & $22.50^{b} \pm 5.00$ & $32.50^{c} \pm 5.00$ & $45.00^{d} \pm 5.77$ & $57.50^{e} \pm 5.00$ & $5.00^{a} \pm 5.77$ \\
\hline$F=51.914$ & & $p$ value $=.000$ & & $\mathrm{df}=5,18$ & \\
\hline \multicolumn{6}{|c|}{ Paeciolmyces lilacinus } \\
\hline $10.00^{a, b} \pm 0.00$ & $10.00^{a, b} \pm 0.00$ & $15.00^{b, c} \pm 5.77$ & $15.00^{b, c} \pm 5.77$ & $17.50^{c} \pm 5.00$ & $5.00^{a} \pm 5.77$ \\
\hline \multirow[t]{2}{*}{$F=4.040$} & & $p$ value $=.012$ & & $d f=5,18$ & \\
\hline & & \multicolumn{4}{|c|}{ Mean mortality $\%$ of 1 -day-old pupae } \\
\hline \multicolumn{6}{|c|}{ Metarhizium anisopliae } \\
\hline $1 \times 10^{6}$ & $1 \times 10^{7}$ & $1 \times 10^{8}$ & $1 \times 10^{9}$ & $1 \times 10^{10}$ & Control \\
\hline $35.00^{b} \pm 5.77$ & $45.00^{c} \pm 5.77$ & $55.00^{d} \pm 5.77$ & $60.00^{d} \pm 8.16$ & $62.50^{d} \pm 5.00$ & $5.00^{a} \pm 5.77$ \\
\hline$F=49.533$ & & $p$ value $=.000$ & & $d f=5,18$ & \\
\hline \multicolumn{6}{|c|}{ Beauveria bassiana } \\
\hline $30.00^{b} \pm 0.00$ & $35.00^{b, c} \pm 5.77$ & $40.00^{c} \pm 0.00$ & $50.00^{d} \pm 8.16$ & $57.50^{d} \pm 5.00$ & $5.00^{a} \pm 5.77$ \\
\hline$F=50.684$ & & $p$ value $=.000$ & & $d f=5,18$ & \\
\hline \multicolumn{6}{|c|}{ Paecilomyces lilacinus } \\
\hline $10.00^{a, b} \pm 0.00$ & $10.00^{a, b} \pm 0.00$ & $15.00^{b} \pm 5.77$ & $12.50^{b} \pm 5.00$ & $15.00^{b} \pm 5.77$ & $5.00^{a} \pm 5.77$ \\
\hline \multirow[t]{2}{*}{$F=2.760$} & & $p$ value $=.051$ & & $d f=5,18$ & \\
\hline & & \multicolumn{4}{|c|}{ Mean mortality\% of 5-day-old pupae } \\
\hline \multicolumn{6}{|c|}{ Metarhizium anisopliae } \\
\hline $1 \times 10^{6}$ & $1 \times 10^{7}$ & $1 \times 10^{8}$ & $1 \times 10^{9}$ & $1 \times 10^{10}$ & Control \\
\hline $25.00^{b} \pm 5.77$ & $35.00^{c} \pm 5.77$ & $45.00^{d} \pm 5.77$ & $45.00^{d} \pm 5.77$ & $77.50^{e} \pm 5.00$ & $5.00^{a} \pm 5.77$ \\
\hline$F=73.174$ & & $p$ value $=.000$ & & $d f=5,18$ & \\
\hline \multicolumn{6}{|c|}{ Beauveria bassiana } \\
\hline $30.00^{b} \pm 0.00$ & $35.00^{b} \pm 5.77$ & $32.50^{b} \pm 5.00$ & $32.50^{b} \pm 5.00$ & $65.00^{c} \pm 5.77$ & $5.00^{a} \pm 5.77$ \\
\hline$F=58.267$ & & $p$ value $=.000$ & & $d f=5,18$ & \\
\hline \multicolumn{6}{|c|}{ Paecilomyces lilacinus } \\
\hline $15.00^{b} \pm 5.77$ & $15.00^{b} \pm 5.77$ & $15.00^{b} \pm 5.77$ & $20.00^{b} \pm 0.00$ & $22.50^{b} \pm 5.00$ & $5.00^{a} \pm 5.77$ \\
\hline \multirow[t]{2}{*}{$F=5.463$} & & $p$ value $=.003$ & & $\mathrm{df}=5,18$ & \\
\hline & & & Mean mortalit) & -old pupae & \\
\hline \multicolumn{6}{|c|}{ Metarhizium anisopliae } \\
\hline $1 \times 10^{6}$ & $1 \times 10^{7}$ & $1 \times 10^{8}$ & $1 \times 10^{9}$ & $1 \times 10^{10}$ & Control \\
\hline $15.00^{b} \pm 5.77$ & $22.50^{b, c} \pm 5.00$ & $27.50^{c} \pm 5.00$ & $37.50^{d} \pm 5.00$ & $45.00^{d} \pm 5.77$ & $5.00^{a} \pm 5.77$ \\
\hline$F=29.286$ & & $p$ value $=.000$ & & $\mathrm{df}=5,18$ & \\
\hline \multicolumn{6}{|c|}{ Beauveria bassiana } \\
\hline $22.50^{b} \pm 5.00$ & $25.00^{b, c} \pm 5.77$ & $25.00^{b, c} \pm 5.77$ & $32.50^{c} \pm 5.00$ & $47.50^{d} \pm 5.00$ & $5.00^{a} \pm 5.77$ \\
\hline$F=26.314$ & & $p$ value $=.000$ & & $d f=5,18$ & \\
\hline \multicolumn{6}{|c|}{ Paecilomyces lilacinus } \\
\hline $12.50^{a, b} \pm 5.00$ & $12.50^{a, b} \pm 5.00$ & $12.50^{a, b} \pm 5.00$ & $15.00^{b, c} \pm 5.77$ & $22.50^{c} \pm 5.00$ & $5.00^{a} \pm 5.77$ \\
\hline$F=4.560$ & & $p$ value $=.007$ & & $\mathrm{df}=5,18$ & \\
\hline
\end{tabular}


Table 2 Toxicity of Metarhizium anisopliae, Beauveria bassiana and Paecilomyces lilacinus to different stages of Ceratitis capitata

\begin{tabular}{|c|c|c|c|c|c|c|c|}
\hline Stages & $\mathrm{LC}_{10}$ Conidia/ml & $\mathrm{LC}_{30}$ Conidia/ml & $\mathrm{LC}_{50}$ Conidia/ml & $\mathrm{LC}_{90}$ Conidia/ml & Slope \pm SE & $x^{2}$ & $p$ \\
\hline \multicolumn{8}{|c|}{ Metarhizium anisopliae } \\
\hline Third larval instar & $9.81 \times 10^{4}$ & $2.53 \times 10^{7}$ & $1.18 \times 10^{9}$ & $1.42 \times 10^{13}$ & $0.314 \pm 0.068$ & 3.284 & 1.000 \\
\hline 1-day-old pupae & $4.09 \times 10^{10}$ & $7.08 \times 10^{4}$ & $6.10 \times 10^{7}$ & $9.10 \times 10^{14}$ & $0.179 \pm 0.064$ & 2.871 & 1.000 \\
\hline 5-day-old pupae & $1.62 \times 10^{4}$ & $4.83 \times 10^{6}$ & $2.49 \times 10^{8}$ & $3.83 \times 10^{12}$ & $0.306 \pm 0.067$ & 6.181 & .995 \\
\hline 8-day-old pupae & $6.66 \times 10^{4}$ & $1.54 \times 10^{8}$ & $3.27 \times 10^{10}$ & $1.61 \times 10^{16}$ & $0.225 \pm 0.069$ & 2.375 & 1.000 \\
\hline \multicolumn{8}{|l|}{ Beauveria bassiana } \\
\hline Third larval instar & $1.86 \times 10^{5}$ & $5.22 \times 10^{7}$ & $2.59 \times 10^{9}$ & $3.62 \times 10^{13}$ & $0.309 \pm 0.069$ & 2.296 & 1.000 \\
\hline 1-day-old pupae & $1.06 \times 10^{2}$ & $1.55 \times 10^{6}$ & $1.18 \times 10^{9}$ & $1.32 \times 10^{16}$ & $0.182 \pm 0.064$ & 1.672 & 1.000 \\
\hline 5-day-old pupae & $2.54 \times 10^{2}$ & $4.62 \times 10^{6}$ & $4.11 \times 10^{9}$ & $6.65 \times 10^{16}$ & $0.178 \pm 0.065$ & 7.813 & .981 \\
\hline 8-day-old pupae & $2.61 \times 10^{3}$ & $9.88 \times 10^{7}$ & $1.46 \times 10^{11}$ & $8.19 \times 10^{18}$ & $0.165 \pm 0.067$ & 3.463 & 1.000 \\
\hline \multicolumn{8}{|c|}{ Paecilomyces lilacinus } \\
\hline Third larval instar & $1.54 \times 10^{6}$ & $2.07 \times 10^{14}$ & $8.84 \times 10^{19}$ & $5.08 \times 10^{33}$ & $0.093 \pm 0.080$ & 2.338 & 1.000 \\
\hline 1-day-old pupae & $8.46 \times 10^{5}$ & $1.97 \times 10^{18}$ & $7.23 \times 10^{26}$ & $6.17 \times 10^{47}$ & $0.061 \pm 0.081$ & 2.689 & 1.000 \\
\hline 5-day-old pupae & $3.89 \times 10^{3}$ & $2.35 \times 10^{13}$ & $1.40 \times 10^{20}$ & $5.05 \times 10^{36}$ & $0.077 \pm 0.074$ & 3.000 & 1.000 \\
\hline 8-day-old pupae & $3.47 \times 10^{5}$ & $2.77 \times 10^{13}$ & $8.22 \times 10^{18}$ & $1.95 \times 10^{32}$ & $0.096 \pm 0.077$ & 3.850 & 1.000 \\
\hline
\end{tabular}

Table 3 Mean percentage of mortality ( \pm SD) of Ceratitis capitata adults at different treatment times (h) using different concentrations of Metarhizium anisopliae, Beauveria bassiana, and Paecilomyces lilacinus

\begin{tabular}{|c|c|c|c|c|c|c|c|c|}
\hline $\mathrm{H}$ & Control & $\begin{array}{l}1 \times 10^{6} \\
\text { Conidia/ml }\end{array}$ & $\begin{array}{l}1 \times 10^{7} \\
\text { Conidia/ml }\end{array}$ & $\begin{array}{l}1 \times 10^{8} \\
\text { Conidia/ml }\end{array}$ & $\begin{array}{l}1 \times 10^{9} \\
\text { Conidia/ml }\end{array}$ & $\begin{array}{l}1 \times 10^{10} \\
\text { Conidia/ml }\end{array}$ & $F$ & $p$ value \\
\hline \multicolumn{9}{|c|}{ Metarhizium anisopliae } \\
\hline 24 & $0.00 \pm 0.00^{a}$ & $10.00 \pm 0.00^{b}$ & $2.50 \pm 5.00^{a}$ & $15.00 \pm 5.77^{b, c}$ & $12.50 \pm 5.00^{b}$ & $20.00 \pm 0.00^{c}$ & 16.560 & 0.000 \\
\hline 48 & $5.00 \pm 5.77^{a}$ & $7.50 \pm 5.00^{a}$ & $20.00 \pm 0.00^{b, c}$ & $25.00 \pm 5.77^{c}$ & $15.00 \pm 5.77^{b}$ & $32.50 \pm 5.00^{d}$ & 17.600 & 0.000 \\
\hline 72 & $0.00 \pm 0.00^{a}$ & $30.00 \pm 0.00^{c}$ & $27.50 \pm 5.00^{c}$ & $30.00 \pm 0.00^{c}$ & $20.00 \pm 0.00^{b}$ & $30.00 \pm 0.00^{c}$ & 135.400 & 0.000 \\
\hline 96 & $10.00 \pm 0.00^{a}$ & $32.50 \pm 5.00^{c}$ & $35.00 \pm 5.77^{c}$ & $20.00 \pm 0.00^{b}$ & $20.00 \pm 0.00^{b}$ & $17.50 \pm 5.00^{b}$ & 25.920 & 0.000 \\
\hline 120 & $15.00 \pm 5.77^{c}$ & $15.00 \pm 5.77^{c}$ & $15.00 \pm 5.77^{c}$ & $7.50 \pm 5.00^{b}$ & $20.00 \pm 0.00^{c}$ & $0.00 \pm 0.00^{a}$ & 9.800 & 0.000 \\
\hline 144 & $0.00 \pm 0.00^{a}$ & $0.00 \pm 0.00^{a}$ & $0.00 \pm 0.00^{a}$ & $0.00 \pm 0.00^{a}$ & $12.50 \pm 5.00^{b}$ & $0.00 \pm 0.00^{a}$ & 25.000 & 0.000 \\
\hline \multicolumn{9}{|c|}{ Beauveria bassiana } \\
\hline 24 & $0.00 \pm 0.00^{a}$ & $0.00 \pm 0.00^{a}$ & $7.50 \pm 9.57^{a}$ & $2.50 \pm 5.00^{a}$ & $22.50 \pm 5.00^{b}$ & $25.00 \pm 5.77^{b}$ & 17.629 & 0.000 \\
\hline 48 & $5.00 \pm 5.77^{a}$ & $12.50 \pm 5.00^{a}$ & $22.50 \pm 5.00^{b}$ & $22.50 \pm 5.00^{b}$ & $22.50 \pm 5.00^{b}$ & $35.00 \pm 5.77^{c}$ & 15.120 & 0.000 \\
\hline 72 & $0.00 \pm 0.00^{a}$ & $0.00 \pm 0.00^{a}$ & $0.00 \pm 0.00^{a}$ & $0.00 \pm 0.00^{a}$ & $0.00 \pm 0.00^{a}$ & $12.50 \pm 5.00^{b}$ & 25.000 & 0.000 \\
\hline 96 & $10.00 \pm 0.00^{a}$ & $22.50 \pm 9.57^{b, c}$ & $15.00 \pm 5.77^{a, b}$ & $27.50 \pm 5.00^{c}$ & $22.50 \pm 5.00^{b, c}$ & $10.00 \pm 0.00^{a}$ & 7.343 & 0.001 \\
\hline 120 & $15.00 \pm 5.77^{a, b}$ & $25.00 \pm 5.77^{b, c}$ & $25.00 \pm 12.91^{b, c}$ & $22.50 \pm 5.00^{b, c}$ & $30.00 \pm 0.00^{c}$ & $7.50 \pm 5.00^{a}$ & 5.647 & 0.003 \\
\hline 144 & $0.00 \pm 0.00^{a}$ & $35.00 \pm 10.00^{c}$ & $30.00 \pm 8.16^{c}$ & $17.50 \pm 5.00^{b}$ & $0.00 \pm 0.00^{a}$ & $2.50 \pm 5.00^{a}$ & 27.323 & 0.000 \\
\hline \multicolumn{9}{|c|}{ Paecilomyces lilacinus } \\
\hline 24 & $0.00 \pm 0.00^{a}$ & $2.50 \pm 5.00^{a}$ & $5.00 \pm 5.77^{a, b}$ & $10.00 \pm 0.00^{b}$ & $20.00 \pm 0.00^{c}$ & $17.50 \pm 5.00^{c}$ & 19.200 & 0.000 \\
\hline 48 & $5.00 \pm 5.77^{a}$ & $10.00 \pm 0.00^{a, b}$ & $10.00 \pm 0.00^{a, b}$ & $15.00 \pm 5.77^{b, c}$ & $17.50 \pm 5.00^{c}$ & $20.00 \pm 0.00^{c}$ & 8.127 & 0.000 \\
\hline 72 & $0.00 \pm 0.00$ & $20.00 \pm 0.00$ & $20.00 \pm 0.00$ & $30.00 \pm 0.00$ & $30.00 \pm 0.00$ & $30.00 \pm 0.00$ & - & - \\
\hline 96 & $10.00 \pm 0.00$ & $20.00 \pm 0.00$ & $20.00 \pm 0.00$ & $20.00 \pm 0.00$ & $20.00 \pm 0.00$ & $30.00 \pm 0.00$ & - & - \\
\hline 120 & $15.00 \pm 5.77^{b}$ & $12.50 \pm 5.00^{b}$ & $15.00 \pm 5.77^{b}$ & $25.00 \pm 5.77^{c}$ & $20.00 \pm 0.00^{b, c}$ & $2.50 \pm 5.00^{a}$ & 9.200 & 0.000 \\
\hline 144 & $0.00 \pm 0.00$ & $0.00 \pm 0.00$ & $0.00 \pm 0.00$ & $0.00 \pm 0.00$ & $0.00 \pm 0.00$ & $0.00 \pm 0.00$ & - & - \\
\hline
\end{tabular}


Table 4 Lethal time LT (h) for Ceratitis capitata treated with different concentrations of Metarhizium anisopliae, Beauveria bassiana, and Paecilomyces lilacinus

\begin{tabular}{|c|c|c|c|c|c|c|c|}
\hline $\begin{array}{l}\text { Concentration } \\
\text { Conidia/ml }\end{array}$ & $\mathrm{LT}_{25}$ & $\mathrm{LT}_{50}$ & $\mathrm{LT}_{75}$ & $\mathrm{LT}_{90}$ & Slope \pm SE & $x^{2}$ & $\mathrm{p}$ \\
\hline \multicolumn{8}{|c|}{ Metarhizium anisopliae } \\
\hline $1 \times 10^{6}$ & 45.083 & 64.390 & 91.967 & 126.755 & $4.357 \pm 0.470$ & 17.398 & .741 \\
\hline $1 \times 10^{7}$ & 49.929 & 64.182 & 82.502 & 103.424 & $6.185 \pm 0.698$ & 28.908 & .147 \\
\hline $1 \times 10^{8}$ & 33.704 & 49.332 & 72.208 & 101.741 & $4.077 \pm 0.447$ & 12.463 & .947 \\
\hline $1 \times 10^{9}$ & 41.718 & 63.467 & 96.555 & 140.859 & $3.701 \pm 0.424$ & 13.909 & .905 \\
\hline $1 \times 10^{10}$ & 29.356 & 40.922 & 57.046 & 76.926 & $4.675 \pm 0.530$ & 7.440 & .998 \\
\hline \multicolumn{8}{|c|}{ Beauveria bassiana } \\
\hline $1 \times 10^{6}$ & 75.240 & 100.199 & 133.439 & 172.688 & $5.421 \pm 0.697$ & 25.201 & .288 \\
\hline $1 \times 10^{7}$ & 50.084 & 79.337 & 125.678 & 190.137 & $3.376 \pm 0.429$ & 37.434 & .021 \\
\hline $1 \times 10^{8}$ & 56.669 & 83.214 & 122.193 & 172.670 & $4.043 \pm 0.501$ & 24.617 & .316 \\
\hline $1 \times 10^{9}$ & 31.858 & 53.344 & 89.319 & 142.045 & $3.013 \pm 0.379$ & 21.962 & .462 \\
\hline $1 \times 10^{10}$ & 23.271 & 41.498 & 74.001 & 124.547 & $2.685 \pm 0.367$ & 4.944 & 1.000 \\
\hline \multicolumn{8}{|c|}{ Paecilomyces lilacinus } \\
\hline $1 \times 10^{6}$ & 61.611 & 98.800 & 158.438 & 242.359 & $3.289 \pm 0.470$ & 7.033 & .999 \\
\hline $1 \times 10^{7}$ & 55.873 & 91.294 & 149.170 & 232.063 & $3.163 \pm 0.436$ & 8.145 & .997 \\
\hline $1 \times 10^{8}$ & 42.199 & 61.589 & 89.889 & 126.328 & $4.108 \pm 0.475$ & 10.356 & .961 \\
\hline $1 \times 10^{9}$ & 31.626 & 49.303 & 76.861 & 114.620 & $3.498 \pm 0.483$ & 6.632 & .980 \\
\hline $1 \times 10^{10}$ & 33.788 & 47.665 & 67.242 & 91.651 & $4.513 \pm 0.488$ & 16.125 & .810 \\
\hline
\end{tabular}

the performances of $M$. anisopliae and B. bassiana induced higher mortality than $P$. lilacinusis.

Sub-lethal concentrations; $\mathrm{LC}_{10}, \mathrm{LC}_{30}$, and lethal concentrations; $\mathrm{LC}_{50}$ and $\mathrm{LC}_{90}$ of the $3 \mathrm{EPF}$ showed that $M$. anisopliae ranked first, causing mortality to late third instar larvae followed by B. bassiana. P. lilacinus, which showed the weakest pathogenic activity against the larval stage (Table 2).

Generally, it was recorded that EPF vary in their pathogenicity on insects and other arthropods. For instance, the pathogenicity of four species of EPF: B. bassiana, M. anisopliae, M. flavoviride, and Paecilomyces fumosoroseus, to various developmental stages of Rhipicephalus sanguineus (Acari: Ixodidae) ticks was compared under laboratory conditions. The most virulent isolate, M. anisopliae-108, caused 92-96\% mortality (Samish et al. 2001). Similarily, the pathogenicity of 13 isolates of $M$. anisopliae and 2 isolates of B. bassiana to C. capitata and C. var. rosa fasciventris showed fluctuation in the mortality rates among larvae (Ekesi et al. 2002).

\section{Susceptibility of C. capitata pupae}

The EPF $M$. anisopliae appeared superior in causing pathogenicity recording the highest mean percentages of mortality in 1-day-old pupae. Using the concentrations ranging between $1 \times 10^{6}$ and $1 \times 10^{10}$ conidia/ml, the mortality rate ranged $35-62.5 \%$, while $B$. bassiana caused 30-57\% pupal mortality. Both fungi increased the mortality in pupae by increasing concentration (Table 1). However, P. lilacinus, showed the least percentages of pupal mortality which ranged (10-15\%) without any effect of fungal concentration increase on pupal mortality (Table 1 ).

The 5-day-old pupae were susceptible to all tested fungi. Metarhizium anisopliae achieved (25 and 77.50\%) pupal mortality at the concentrations $1 \times 10^{6}$ and $1 \times$ $10^{10}$ conidia $/ \mathrm{ml}$, respectively. While $B$. bassiana treated pupae recorded $30-65 \%$ mortality. Unlike to other developmental stages (third larval instar and 1-day-old pupa), $P$. lilacinus showed slightly more activity at 5day-old pupae and resulted (15 and $22.50 \%$ ) pupal mortality. In 5-day-old pupae, all fungi increased mortality as the concentration increases (Table 1).

Similar pattern to those 5-day-old pupae was obtained while using 8-day-old pupae, showed significant differences $(p$ value $<.05)$ in the mean percentages of mortality among different concentrations. The fungus $M$. anisopliae showed pupal mortality between 15 and $45 \%$ at the concentrations $\left(1 \times 10^{6}\right.$ and $1 \times 10^{10}$ conidia $\left./ \mathrm{ml}\right)$ while B. bassiana and P. lilacinus caused $22.5-47.5 \%$ and $12.5-22.5 \%$ pupal mortality, respectively.

Comparing the effect of equal concentrations of EPF on different ages of pupae, the mortality rates were compared at the same concentration (Fig. 1b-d). On 1- and 5-day-old pupae (Fig. 1b, c), the 


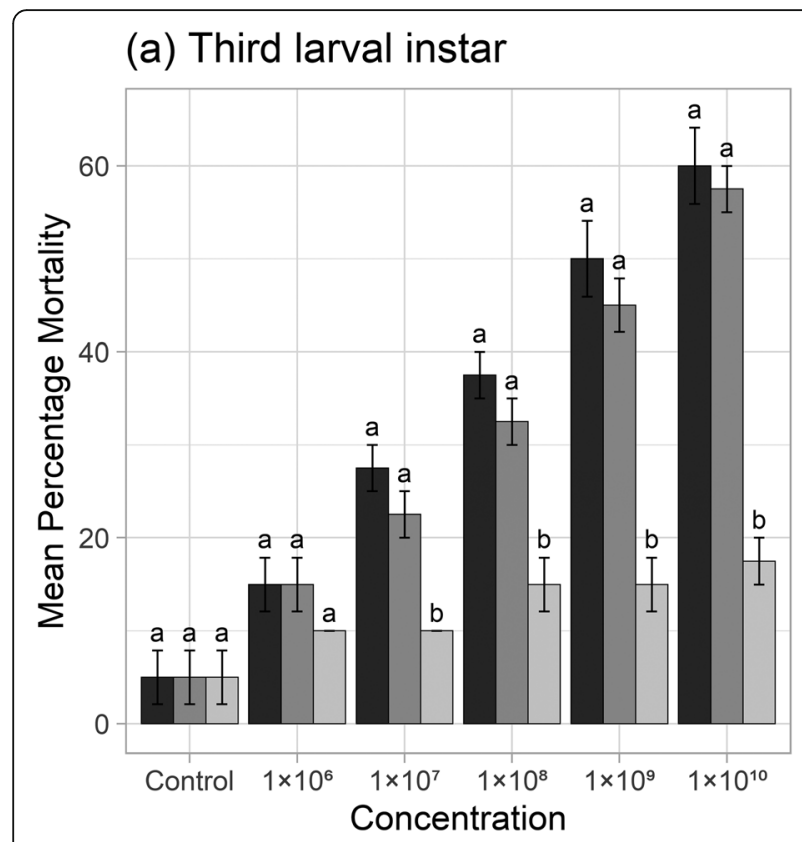

Fungus M.anisopliae $\square$ B.bassiana $\square$ P.lilacinus

\section{(b) One-day old pupae}

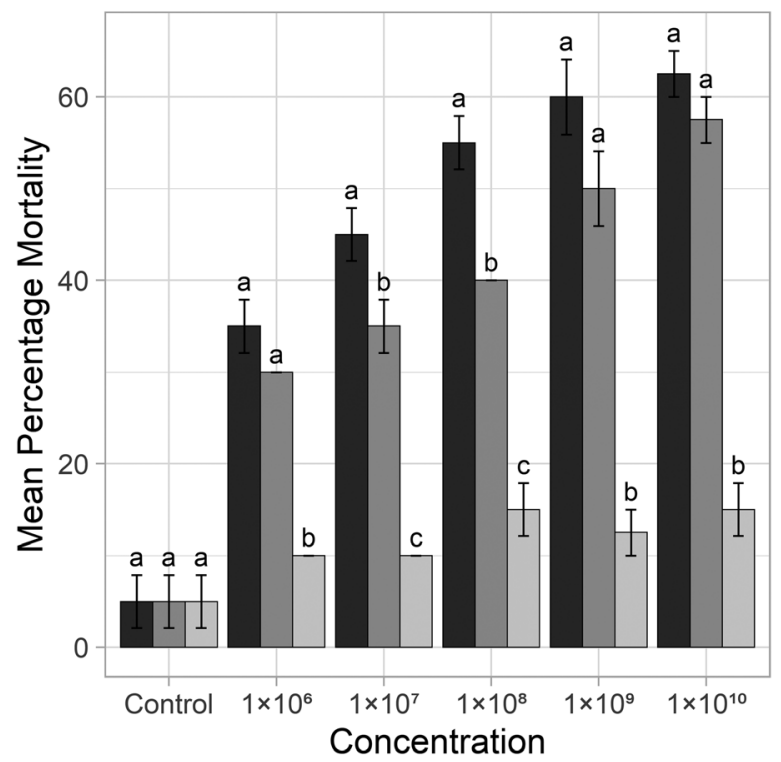

Fungus M.anisopliae $\square$ B.bassiana $\square$ P.lilacinus (c) Five-day old pupae

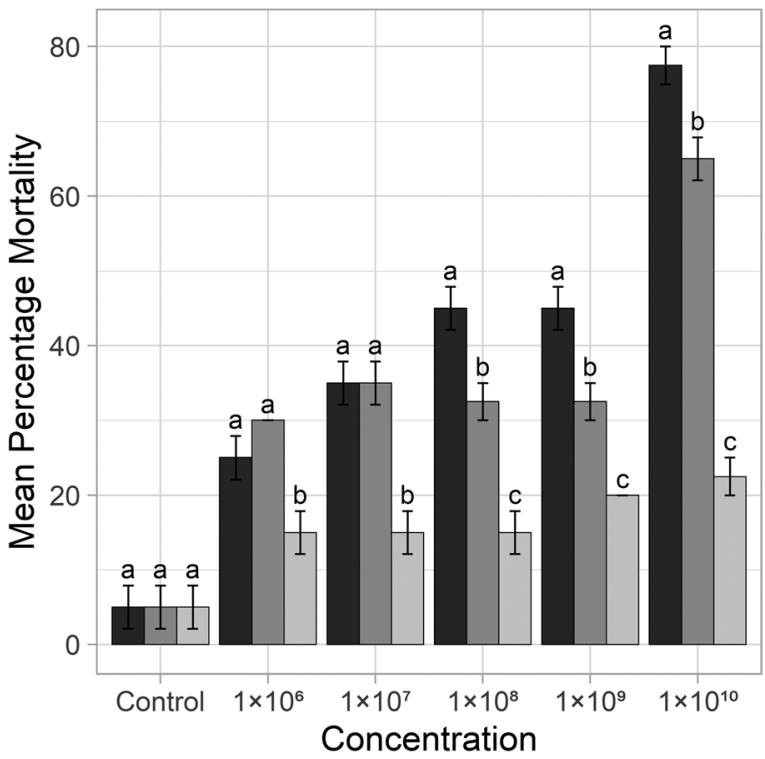

Fungus M.anisopliae $\square$ B.bassiana $\square$ P.lilacinus

\section{(d) Eight-day old pupae}

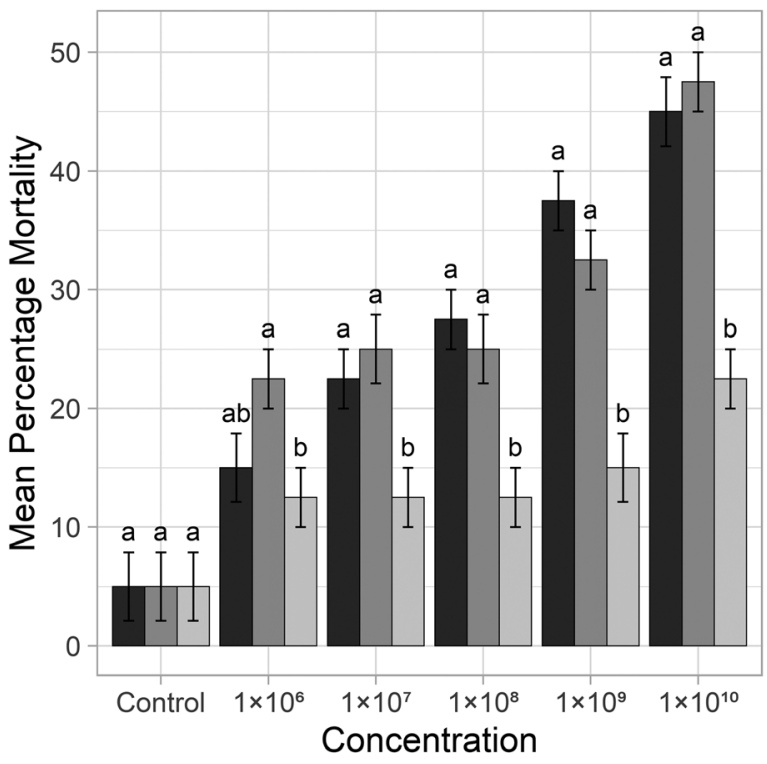

Fungus $\square$ M.anisopliae $\square$ B.bassiana $\square$ P.lilacinus

Fig. 1 Mean percentage mortality (\% \pm SD) in immature stages of Ceratitis capitata treated with different concentrations of Metarhizium anisopliae, Beauveria bassiana, and Paecilomyces lilacinus. a Late third larval instar. b 1-day-old pupae. c 5-day-old pupae. d 8-day-old pupae. Bars with the same letter are not significantly different at $p>0.05$

performances of $M$. anisopliae, B. bassiana, and $P$. lilacinus, showed similar pattern but the difference between $M$. anisopliae and B. bassiana and P. lilacinus was clear at all concentrations giving the highest pathogenicity to $M$. anisopliae followed by $B$. bassiana and finally P. lilacinus (Fig. 1b, c).

For 8-day-old pupae (Fig. 1d), the pattern of pathogenicity was very similar to that of larvae (Fig. 1a) where 
there was no differences between all treatments only at the lowest concentration, $1 \times 10^{6}$ (Fig. 1d). However, at the highest concentrations, the performances of $M$. anisopliae and B. bassiana caused higher mortality than $P$. lilacinusis. Similar results were obtained when analyzing sub-lethal concentrations; $\mathrm{LC}_{10}, \mathrm{LC}_{30}$, and lethal concentrations; $\mathrm{LC}_{50}$ and $\mathrm{LC}_{90}$ of the $3 \mathrm{EPF}$ which also revealed that $M$. anisopliae ranked first in its pathogenicity to 1-, 5-, and 8-day-old pupae, followed by B. bassiana and P.lilacinus, which showed the weakest pathogenic activity against the mentioned stages (Table 2).

Oreste et al. (2015) studied the interaction between age of puparia (at 2, 4, 6 days old) and fungal strains. They addressed both the emergence of $C$. capitata adults and the mortality of pupae. They found that the fungal treatments of different $B$. bassiana strains was higher on 2-day-old puparia (49.16 and 51.33\% of mycosed puparia for ATCC 74040 and AL1 strain, respectively), while the rate of mycoses was low and ranged between 39 and $27.16 \%$, when fungal treatments were performed on 4- and 6-day-old puparia. LozanoTovar et al. (2013) found significant differences among strains of Beauveria spp. and Metarhizium spp. in the total percentage of non-viable puparia of $C$. capitata and non-viable puparia showing fungal outgrowth, with percentages ranged from 5.0 to $45.0 \%$. Quesada-Moraga et al. (2006) tested the pathogenicity of 10 strains of $B$. bassiana and 5 of $M$. anisopliae against puparia and adults of $C$. capitata, finding that only 2 strains of $B$. bassiana and one of $M$. anisopliae caused mortality higher than $50 \%$, when puparia were immersed in the conidial suspensions. Imoulan et al. (2011) tested several $B$. bassiana strains against medfly pupae, finding that when insects were exposed to $10^{8}$ conidia/ml, the adult emergence ranged from 0 to $23.33 \%$ after 10 days posttreatment.

\section{Susceptibility of $C$. capitata flies to entomopathogenic fungi}

The three fungal species were pathogenic to the flies; however, it was difficult to identify a clear pattern of mortality either with time after treatments by concentrations of the used fungi (Table 3). Mortality of $C$. capitata adults by all of the used fungi showed fluctuated increases and decreases with time when compared to control (Table 3). Similar limitation was achieved while calculating the lethal time where the lethal time to kill $25 \%\left(\mathrm{LT}_{25}\right), 50 \%\left(\mathrm{LT}_{50}\right), \quad 75 \%$ $\left(\mathrm{LT}_{75}\right)$, and $90 \%\left(\mathrm{LT}_{90}\right)$ varied among the three fungal isolates (Table 4).

The fluctuation in mortality lethal time and concentration among adults might be linked to the method of treatment where adults were kept in 0.51 plastic cage in which only $2 \mathrm{ml}$ of conidial suspension was sprayed.
Based on the fact that they can fly away from the contaminated area, adult might avoid infection with the fungi. The red palm weevil adults treated with $B$. bassiana and $M$. anisopliae showed different lethal time by changing the spray method between dry powder and aqueous suspension (Gindin et al. 2006). Beris et al. (2013) verified the pathogenicity of several strains of $B$. bassiana, Isaria fumosorosea Wize-formerly Paecilomyces fumosoroseus (Wize) Brown et Smith and M. anisopliae under laboratory conditions against pupal and adult stages of Mediterranean fruit fly via different exposure routes. Castillo and Moya (2000) had confirmed the susceptibility of adults of $C$. capitata to infection by several isolates of EPF, especially, M.anidopliae and $P$. fumosoroseus CG-260, which 6 days after treatments, exhibited similar levels of activity, being significantly more pathogenic than the other fungi. Thus, at the two highest concentrations, non-significant differences were found, while at the lowest concentrations, $M$. anisopliae was slightly more virulent than P. fumosoroseus CG-260. Ten days after treatment, the mortality caused by the fungi increased, mainly at the three highest concentrations. M. anisopliae and P. fumosoroseus CG260 were the most pathogenic fungi. In general, the speed of mortality of the host is correlated positively with conidial concentration (Fargues and Rodriguez-Rueda 1980). In this work, time-mortality responses could be useful to determine specific clues of fungal-host interactions. The application of conidial preparations to natural soil reduced insect emergence and the adult life span and represents a promising strategy for fruit fly integrated management.

The present findings are in agreement with many works who confirmed the susceptibility of other tephritids to infection with $M$. anisopliae and B.bassiana (Dimbi et al. 2003; Quesada-Moraga et al. 2006; Ladurner et al. 2008). Current research in controlling fruit fly and other harmful pests is highly focusing on searching for safe biopesticides and other safe alternative methods of control (Ali and Ibrahim 2018; Ibrahim and Ali 2018). Such safe biopesticides as well EPF are believed to serve as promising tools in pest management rather than chemical insecticides.

\section{Conclusion}

Entomopathogenic fungi, as soil-borne micro-organisms, are one of the most important agent in biological control programs against $C$. capitata. Three species of fungi (local strains) were tested against the adults and different immature stages (larvae and pupae) of C. capitata to evaluate their pathogenicity. Our results suggest $M$. anisopliae and $B$. bassiana have a great value in its virulence to C. capitata. 


\section{Abbreviations}

IPM: Integrated pest management; EPF: Entomopathogenic fungi; PDA: Potato dextrose agar; RH: Relative humidity; ANOVA: Analysis of variance; LC: Lethal concentration; LT: Lethal time; SD: Standard deviation

\section{Acknowledgements}

The authors are grateful to all technical staff and researchers at the Center of Mycology, Assiut University for supplying fungal strains.

\section{Authors' contributions}

NAS reared C. capitata and put strategy to achieve this work. SMA achieved this investigation. NAS and SMA are the contributors in writing the manuscript. AMAI and AMA revised the manuscript. All authors read and approved the final manuscript.

\section{Funding}

Plant Protection Research Institute, ARC, Egypt, and Faculty of Science, Assiut University.

\section{Availability of data and materials}

All data and materials are available.

\section{Consent for publication}

Not applicable

\section{Competing interests}

Not applicable

\section{Author details}

${ }^{1}$ Plant protection research institute, ARC, Dokki, Giza, Egypt. ${ }^{2}$ Department of Zoology and Entomology, Faculty of Science, Assiut University, Assiut 71516, Egypt

\section{Received: 16 December 2019 Accepted: 16 March 2020}

Published online: 01 May 2020

\section{References}

Abbott WS (1925) A method of computing the effectiveness of an insecticide. J Econ Entomol. 18:265-267

Ali MAM, Ibrahim AMA (2018) Castor and camphor essential oils alter hemocyte populations and induce biochemical changes in larvae of Spodoptera littoralis (Boisduval) (Lepidoptera : Noctuidae). J Asia Pacific Entomol. 21(631-637)

Beris El, Papachristos DP, Fytrou A, Antonatos SA, Kontodimas DC (2013) Pathogenicity of three entomopathogenic fungi on pupae and adults of the Mediterranean fruit fly, Ceratitis capitata (Diptera: Tephritidae). J Pest Sci 86 275-284

Castillo MA, Moya P, Herna'ndez E, Primo-Yu'fera E (2000) Susceptibility of Ceratitis capitata Wiedemann (Diptera: Tephritidae) to entomopathogenic fungi and their extracts. Biol Control 19:274-282

Dimbi S, Maniania NK, Lux SA, Ekesi S, Mueke JK (2003) Pathogenicity of Metarhizium anisopliae (Metsch.) Sorokin and Beauveria bassiana (Balsamo) Vuillemin, to three adult fruit flyspecies: Ceratitis capitata (Weidemann), C. rosa var. fasciventrisKarsch and C. cosyra (Walker) (Diptera: Tephritidae). Mycopathologia 156:375-382

Ekesi S, Maniania NK, Lux SA (2002) Mortality in three African Tephritid fruit fly puparia and adults caused by the entomopathogenic fungi, Metarhizium anisopliae and Beauveria bassiana. Biocontrol Sci Technol 12:7-17

Ekesi S, Maniania NK, Lux SA (2003) Effect of soil temperature and moisture on survival and infectivity of Metarhizium anisopliae to four Tephritid fruit fly puparia. J Invertebr Pathol 83:157-167

El-Sayed AIM (1979) Biotic potential of Ceratitis capitata (Wied.) as influenced by the type of diet. M. Sc. Thesis, Fac. Agric. Zagazig Univ.

Fargues J, Rodriguez-Rueda D (1980) Sensibilite` des larves de Spodoptera littoralis [Lep. Noctuidae] aux hyphomyce'tes entomopathoge 'nes Nomuraea rileyi et Paecilomyces fumosoroseus. Entomophaga 25:43-54

Finney DJ (1971) Probit analysis, 3rd edn. Cambridge University Press, London

Gabarty A, Salem HM, Fouda MA, Abas AA, Ibrahim AA (2014) Pathogenicity induced by entomopathogenic fungi Beauveria bassiana and Metarhizium anisopliae in Agrotisipsilon(Hufn.). J Radiat Res Appl Sci 7:95-100

Gindin G, Levski S, Glazer I et al (2006) Evaluation of the entomopathogenic fungi Metarhizium anisopliae and Beauveria bassiana against the red palm weevil Rhynchophorus ferrugineus. Phytoparasitica 34:370-379 https://doi.org/ 10.1007/BF02981024

Ibrahim AMA, Ali MAM (2018) Silver and zinc oxide nanoparticles induce developmental and physiological changes in the larval and pupal stages of Spodoptera littoralis (Lepidoptera : Noctuidae). J Asia Pacific Entomol. 21: 1373-1378

Imoulan A, Alaoui A, El Meziane A (2011) Natural occurrence of soil-borne entomopathogenic fungi in the Moroccan Endemic forest of Argania spinosa and their pathogenicity to Ceratitis capitata. World J Microbiol Biotechnol 27: 2619-2628

Konstantopoulou MA, Mazomenos BE (2005) Evaluation of Beauveria bassiana and $B$. brongniartii strains and four wild-type fungal species against adults of Bactrocera oleae and Ceratitis capitata. Biocontrol 50:293-305

Lacey LA, Shapiro-Ilan DI (2008) Microbial control of insect pests in temperate orchard systems: potential for incorporation into IPM. Annu Rev Entomol. 53: $121-144$

Ladurner E, Benuzzi M, Fiorentini F, Franceschini S (2008) Beauveria bassiana strain ATCC 74040 (Naturalis ${ }^{\circledR}$ ), a valuable tool for the control of the cherry fruit fly (Rhagoletis cerasi), pp 93-97. In: Boos M (ed) Proceedings of Ecofruit, 13th International Conference on Cultivation Technique and Phytopathological Problems in Organic Fruit-Growing, Weinsberg Lozano-Tovar MD, Ortiz-urquiza A, Garrido-Jurado I, Trapero-Casas A, QuesadaMoraga $E$ (2013) Assessment of entomopathogenic fungi and their extracts against a soil-dwelling pest and soil-borne pathogens of olive. Biological Control 67:409-420 https://doi.org/10.1016/j.biocontrol.2013.09.006

Martinez-Ferrer MT, Campos JM, Fibla JM (2012) Field efficacy of Ceratitis capitata (Diptera: Tephritidae) mass trapping technique on clementine groves in Spain.J. Appl Entomol 136(3):181-190

Oreste M, Baser N, Bubici G, Tarasco E (2015) Effects of Beauveria bassiana strains on the Ceratitis capitata-Psyttalia concolor system. Bull Insectol 68(2):365-272

Papadopoulos NT, Katsoyannos BI, Carey JR, Kouloussis NA (2001) Seasonal and annual occurrence of the Mediterranean fruit fly (Diptera: Tephritidae) in northern Greece. Ann Entomol Soc Am 94:41-50

Perry AS, Yamamoto I, Ishaaya I, Perry RY (1998) Insecticides in agriculture and environment: retrospects and prospects. Springer, Berlin, p 275

Quesada-Moraga E, Navas-Cortés JA, Maranhao EAA, Ortiz-Urquiza A, Santiago-Alvarez C (2007) Factors affecting the occurrence and distribution of entomopathogenic fungi in natural and cultivated soils. Mycol Res 111:947-966

Quesada-Moraga E, Ruiz-Garcia A, Santiago-Ávarez C (2006) Laboratory evaluation of entomopathogenic fungi Beauvaria bassiana and Metarhizium anisopliae against puparia and adults of Ceratitis capitata (Diptera: Tephritidae). J Econ Entomol 99(6):1955-1966

Samish M, Gindin G, Alekseev E, Glazer I (2001) Pathogenicity of entomopathogenic fungi to different developmental stages of Rhipicephalus sanguineus (ACARI: IXODIDAE). J Parasitol 87(6):1355-1359 https://doi.org/10. 1645/0022-3395(2001)087[1355:POEFTD]2.0.CO;2

Soliman NA, Ibrahim AA, Shams El-Deen MM, Shams Ramadan NF, Farag SR (2014) Entomopathogenic nematodes and fungi as bioControl agents for the peach fruit fly, Bactrocera zonata (Saunders) and the Mediterranean fruit fly, Ceratitis capitata (Wiedemann) soil borne-stages. Egypt J Biol Pest Co. 24:497-502

Sookar P (2014) Study of two potential entomopathogenic fungi, Metarhizium anisoplia and Beauveria bassianaa for the biocontrol of fruit flies (Diptera: Tephritidae) of economic importance in Mauritius. PhD. Thesis, Fac.Science. Mauritius Univ.

Stark JD, Vargas R (2009) An evaluation of alternative insecticides to diazinon for control of Tephritid fruit flies (Diptera: Tephritidae) in soil. J Econ Entomol 102:139-143

Tanaka N, Steiner LF, Ohinata K, Okamoto R (1969) Low cost larval rearing medium for mass rearing production of oriental and Mediterranean fruit flies. J Econ Entomol 62:967-968

Thaczuk C, Krol A, Majchrowska-Safaryan A, Nicewicz L (2014) The occurrence of entomopathogenic fungi in soils from fields cultivated in a conventional and organic system. J Ecol Eng 15:137-144

Uzman D, Pliester J, Leyer I, Entling MH, Reineke A (2019) Drivers of entomopathogenic fungi presence in organic and conventional vineyard soils. Appl Soil Ecol 133:89-97

\section{Publisher's Note}

Springer Nature remains neutral with regard to jurisdictional claims in published maps and institutional affiliations. 